\title{
Mandibular osteomyelitis immediately after implant placement: A case report and review of literature
}

\author{
Chang-Su Kim ${ }^{1,2}$, Dong Uk Park ${ }^{1}$, Ho Lee ${ }^{1,2}$, Yoon-Sic Han ${ }^{1,2}$ \\ Department of Oral and Maxillofacial Surgery, 'SMG-SNU Boramae Medical Center, ${ }^{2}$ School of Dentistry and Dental Research \\ Institute, Seoul National University, Seoul, Korea
}

\begin{abstract}
Mandibular osteomyelitis (OM) is uncommon in the course of implant placement. However, reports of cases of OM caused by implant placement have been steadily increasing. Presented here is the case of a 62-year-old man who developed OM of the mandible immediately after implant placement in a mandibular molar. The patient gradually recovered from the spreading OM following the removal of the implant and prolonged antibiotic therapy. In addition, we review the literature dealing with implant-associated OM. (JOURNAL OF DENTAL IMPLANT RESEARCH 2021;40(3):107-111)
\end{abstract}

Key Words: Dental implant, Osteomyelitis, Antibiotic therapy

\section{INTRODUCTION}

Osteomyelitis $(\mathrm{OM})$ of the jaws is an inflammatory bone disease that originates in the medullary cavity and Haversian systems and spreads outwardly into cortex and periosteum. Bone necrosis leading to sequestration, which is a typical sign of OM, occurs when the inflammation causes vascular compromise in the bone tissue $^{1)}$. OM in the maxillofacial region tends to extend to adjacent tissue mainly by local extension, rather than by a hematogenous route ${ }^{2)}$.

A dental implant as a foreign body is one of the causative factors of $\mathrm{OM}$ in the jaws, along with odontogenic infection, trauma and radiation therapy ${ }^{3-5)}$. A majority of $\mathrm{OM}$ cases are caused by bacterial infection, and the incidence of implant-associated OM is relatively low compared with other causative factors. However, the complications of OM after implant placement can be severe, especially in elderly individuals with preexisting conditions such as diabetes mellitus, immunosuppressive disorders and malnutrition ${ }^{5}$.
Dental implants have been widely used at the edentulous ridge for oral rehabilitation over the past decades, and the incidence of post-implant $\mathrm{OM}$ seems to be increasing $^{6)}$. However, to date, the literature dealing with $\mathrm{OM}$ in the jaws following implant placement has been scarce. Thus, we present a rare case of implant-induced $\mathrm{OM}$ with gradual recovery along with a literature review.

\section{CASE REPORT}

The study was approved by the Institutional Review Board of the Seoul Metropolitan Government- Seoul National University Boramae Medical Center (IRB No. 30-2021-109). The present study was designed in accordance with the Declaration of Helsinki. A 62-year-old man was referred to Seoul Metropolitan Government - Seoul National University Boramae Medical Center in May 2020 with persistent pain and purulent discharge following immediate implant placement in the lower left first molar. The implant had been placed the previous month following a cracked tooth in local dental clinic, Seoul in Korea

Received August 7, 2021, Revised August 23, 2021, Accepted August 31, 2021. (c) Journal of Dental Implant Research.

This is an open access article distributed under the terms of the Creative Commons Attribution Non-Commercial License (http://creativecommons.org/licenses/by-nc/4.0) which permits unrestricted non-commercial use, distribution, and reproduction in any medium, provided the original work is properly cited. 
(Fig. 1). The implant surgery was performed by a prosthodontist with more than 2 years of surgical experience. An osteolytic lesion was detected at the surgical site, and the implant was removed at postoperative 1 month at a local clinic (Fig. 2). Despite these interventions, the patient showed little improvement in pain during the following 2 weeks, and he was referred for further treatment at the Department of Oral and Maxillofacial Surgery in our hospital.

According to the patient's past medical history, he was a smoker with diabetes controlled by Metformin. His fasting blood glucose was $>110 \mathrm{mg} / \mathrm{dL}$. Physical examination showed gingival swelling with purulent discharge and fistula at the buccal gingiva of the first premolar area, tenderness at the surgical site and resin-wire splinted on the canine to the second premolar. Radiographs showed a diffuse radiolucent lesion spread widely on the left side of the mandible (Fig. 3).

A diagnosis of implant-induced OM was made based on clinical and radiographic findings at the first visit, and the patient was informed of the likely necessity of addi-

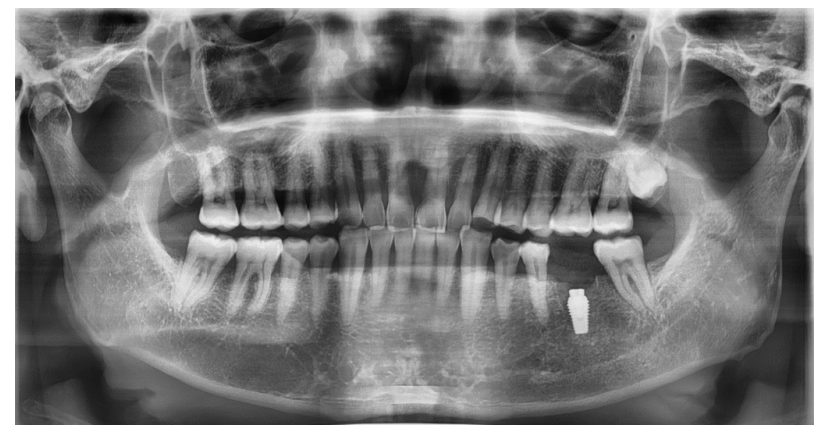

Fig. 1. Panoramic radiograph after immediate implantation of the lower left first molar. tional surgical intervention under general anesthesia if the symptoms persisted. He was treated with a 6-week course of empirical amoxicillin/clavulanic acid, Augmentin (Ilsung Pharmaceutical, Seoul, Korea), $625 \mathrm{mg}$ twice a day orally, on an outpatient basis, with regular follow-up to be performed every 2 weeks for 2 months to monitor for deterioration of the lesion. At the first follow-up visit (2 weeks later), the patient showed significant improvement in pain, and bone biopsy for microorganisms was deferred. At the second visit (4 weeks later), the buccal fistula and purulent discharge in the lower left mandible was cleared, and the patient remained symptom-free without additional surgical intervention. At the 5-month follow-up, the radiological examination showed definite bone regeneration in the lower left mandible (Fig. 4C, 4D). Recurrence of OM was not observed at the 1-year follow-up.

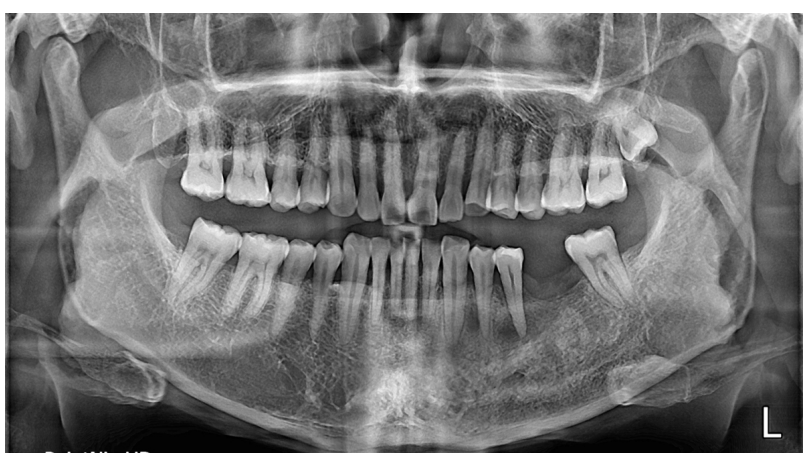

Fig. 2. Panoramic radiograph at postoperative 1 month. An osteolytic lesion is seen extending anteriorly from the site of implant placement to the canine.
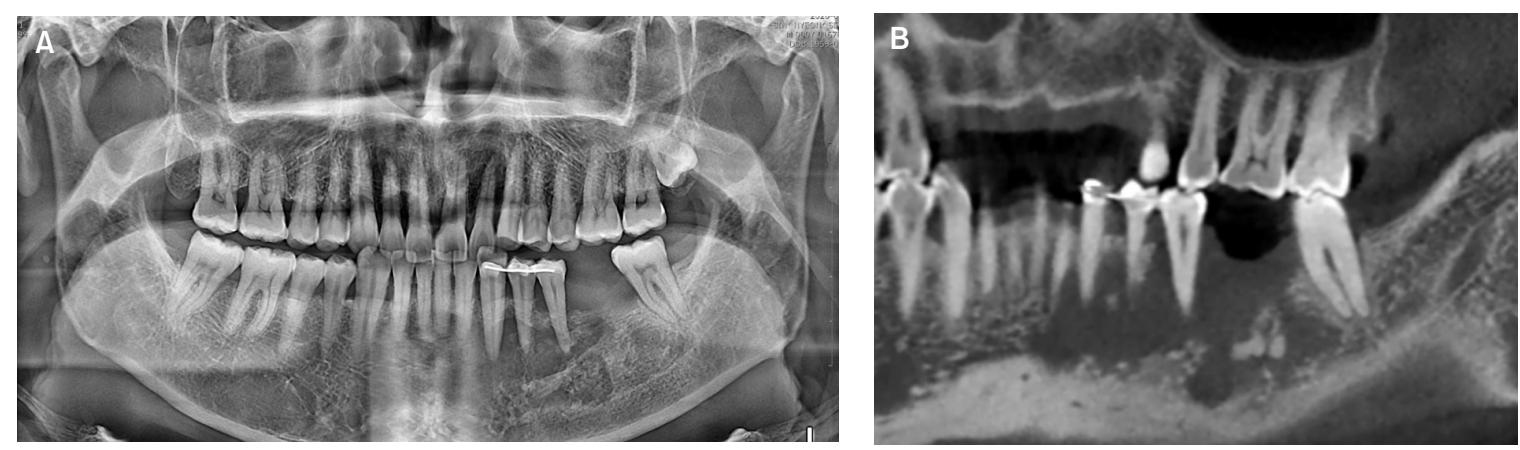

Fig. 3. (A) Panoramic radiograph at postoperative 1.5 months. The implant has been removed. (B) Computed tomography (CT) at postoperative 1.5 months. A diffuse radiolucent lesion extends from the operative site to the lateral incisor. 

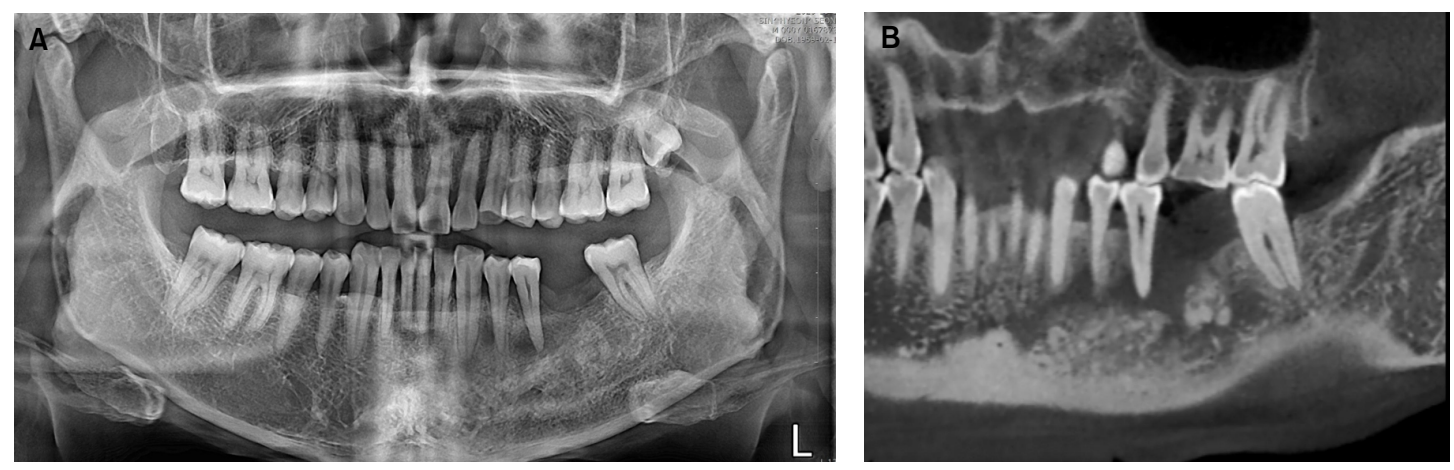

Fig. 4. (A) Panoramic radiograph at the 5-month follow-up. (B) CT scan at the 5-month follow-up. Definite bone regeneration is seen in the lower left mandible.

\section{DISCUSSION}

This report details a case of clinically diagnosed implant-induced OM after immediate implant placement in the posterior mandible. The patient had clinical signs of spreading infection and implant failure in the lower left mandible at 1 month after surgery. Although the implant was removed, the patient's symptoms persisted. Fortunately, he had a gradual recovery with prolonged antibiotics treatment, and at the 1-year follow-up, he was symptom-free and showed definite bone regeneration.

Dental implantation is generally regarded as a reliable method to substitute missing teeth. However, there are several reports of implant-induced OM resulting in devastating consequences involving radical surgery such as segmental or marginal mandibulectomy combined with reconstruction using fibular free flap $(\mathrm{FFF})^{5,7-9)}$. Kesting et al. and Schlund et al. have both reported hemimandibulectomy with FFF reconstruction in cases of recurrent and chronic suppurative $\mathrm{OM}$ after implantation in the mandible ${ }^{7,9)}$. These radical interventions were required in spite of long-term antibiotic treatment and multiple sequestrectomies. Similarly, Doll et al. have reported performing repeated surgeries for refractory cases of implanted-induced $\mathrm{OM}$ before eventually treating patients with partial mandibulectomy and FFF reconstruction followed by long-term administration of intravenous antibiotics $^{10)}$. Chatelain et al. performed partial mandibular resection of infected and necrotic tissue and open reduction and internal fixation with iliac bone graft ${ }^{11)}$. Thus, when the healing process following implant surgery is not on the routine course of recovery, close observation of the implant site is needed to avoid radical surgery, especially in cases of individuals with systemic comorbidities.

The occurrence of implant-associated OM was higher among women, in the mandible and in patients with systemic conditions including diabetes mellitus and in those under bisphosphonate treatment for osteoporosis and cancer ${ }^{6}$. Tobacco use, an immunocompromised state and inexperience with the surgical technique have also been identified as risk factors for $\mathrm{OM}^{12)}$. In the present case, the patient is a smoker and has diabetes mellitus, which could have contributed to the development of OM. We believe that local factors as well as systemic factors can affect the incidence of implant-induced OM. A cracked tooth before implantation is one of the local factors associated with the spread of infection to periapical tissue by a pulpal or periodontal route. Infected periapical remnants after tooth extraction could be a source of bacterial contamination in implant placement procedure. Although immediate implantation itself is known to be a predictable treatment option, even in an endodontically infected site $^{13)}$, the possibility for the occurrence of OM induced by implants may exists unless complete curettage of extraction socket is performed. During implant drilling procedure, bacterial contaminants would invade deep into medullar bone and OM could be established around inserted implant.

The typical treatment of chronic OM consists of a combination of antibiotic therapy and surgical intervention. There is no specific treatment guideline established for implant-induced OM or consensus on whether the involved implants should be removed or left ${ }^{14)}$. However, 
once implant-associated $\mathrm{OM}$ is detected, immediate removal of necrotic tissue as well as implants and long-term antibiotic treatment are essential ${ }^{8,10,11,14,15)}$. Antibiotics therapy is only a palliative treatment when used alone because the penetration of antibiotics is limited by the vascular insufficiency of necrotic bone. Thus, surgical removal of necrotic bone should be indispensable in the management of OM. The effective duration of antibiotic therapy remains controversial, with some studies suggesting 4 to 12 weeks postoperatively ${ }^{16,17)}$. In the present case, antibiotics were orally administered for approximately 8 weeks after implant removal.

Recently the combination of pentoxifylline and tocopherols was also reported to have a positive effect on bony healing process of osteonecrosis of the jaw ${ }^{18)}$. Seo et al. showed favorable bony healing manifesting increased radiographic density in panoramic radiograph when pentoxifylline and tocopherols were used to treat chronic $\mathrm{OM}$ patients for more than 3 months ${ }^{19)}$. This treatment protocol could be adjunctive therapy for chronic OM together with traditional conservative treatment although there are still many aspects to be elucidated such as mechanism, drug dosage and side effects.

Tissue cultures from patients with mandibular OM show polymicrobial infection ${ }^{20,21)}$. Microbes identified among patients with OM associated with dental implants have included Streptococcus, Peptococcus, Peptostreptococcus, Actinomyces, Staphylococcus (including S. aureus) and Candida species ${ }^{6}$. In the present case, since the patient showed improvement in signs and symptoms with empirical antibiotic treatment on the second follow-up, bone biopsy was not needed. However, in case of antibiotics resistance in $\mathrm{OM}$, the antibiotic regimen should be properly tailored according to the results of microbial culture and in consultation with infectious diseases specialists.

In treatment of $\mathrm{OM}$, it is of paramount importance to monitor the patient's prognosis according to treatment. However, conventional diagnostic tools such as plain radiographs (periapical and panoramic) and computed tomography have limitations to identify the subtle change of radiographic density for a short-term treatment. Real-time diagnostic tool, utilizing quantitative light-induced fluorescence (QLF), could be used for the re-evaluation of treatment in OM patients as a complement to ex- isting conventional methods. Kim et al. showed that visual grouping of bone can be possible as bacterial invasive, demineralized, and vital bone respectively by QLF system $^{22}$. QLF system was applicable even for oral soft tissue necrosis ${ }^{23)}$. We consider that this QLF system could help clinicians visually recognize change in the area of infection during follow-up.

\section{CONCLUSION}

A few cases of OM induced by implantation have been reported while implant surgery has become widely performed. In order to prevent implant-induced $\mathrm{OM}$, the removal of possible source of infection in the area of implant placement should be preceded. Regular follow-up is also necessary to detect potential OM in early stage, especially for smokers and patients with systemic condition. Finally, When implant-induced OM appears during follow-up, prolonged antibiotics therapy along with immediate implant removal are required to prevent serious consequences.

\section{CONFLCT OF INTEREST}

The author has no financial interests to declare with regard to the content of this article.

\section{ORCID}

Chang-Su Kim, https://orcid.org/0000-0003-0193-5425 Dong Uk Park, https://orcid.org/0000-0001-8091-7879

Ho Lee, https://orcid.org/0000-0002-0413-2954

Yoon-Sic Han, https://orcid.org/0000-0001-8060-5330

\section{REFERENCES}

1. Neville BW. Oral and maxillofacial pathology. 3rd ed. ed. St. Louis, Mo.: Saunders/Elsevier; 2009.

2. Koorbusch GF, Fotos P, Goll KT. Retrospective assessment of osteomyelitis. Etiology, demographics, risk factors, and management in 35 cases. Oral Surg Oral Med Oral Pathol 1992;74:149-54.

3. Hudson JW. Osteomyelitis of the jaws: A 50-year perspective. J Oral Maxillofac Surg 1993;51:1294-301.

4. Koorbusch GF, Deatherage JR, Curé JK. How Can We Diagnose and Treat Osteomyelitis of the Jaws as Early as Possible? Oral 
Maxillofac Surg Clin North Am 2011;23:557-67.

5. Yahalom R, Ghantous Y, Peretz A, Abu-Elnaaj I. The possible role of dental implants in the etiology and prognosis of osteomyelitis: A retrospective study. Int J Oral Maxillofac Implants 2016;31:1100-9.

6. Kellesarian SV, Javed F, Romanos GE. Osteomyelitis Arising Around Osseointegrated Dental Implants: A Systematic Review. Implant dentistry : ID, the international journal of oral implantation 2018;27:226-35.

7. Schlund M, Raoul G, Ferri J, Nicot R. Mandibular Osteomyelitis Following Implant Placement. J Oral Maxillofac Surg 2017;75: 2560.e1-.e7.

8. Semel G, Wolff A, Shilo D, Akrish S, Emodi O, Rachmiel A. Mandibular osteomyelitis associated with dental implants. A case series. Eur J Oral Implantol 2016;9:435-42.

9. Kesting MR, Thurmüller P, Ebsen M, Wolff K-D. Severe osteomyelitis following immediate placement of a dental implant. Int J Oral Maxillofac Implants 2008;23:137-42.

10. Doll C, Hartwig S, Nack C, Nahles S, Nelson K, Raguse J-D. Dramatic course of osteomyelitis in a patient treated with immediately placed dental implants suffering from uncontrolled diabetes: A case report. Eur J Oral Implantol 2015;8:405-10.

11. Chatelain S, Lombardi T, Scolozzi P. Streptococcus anginosus Dental Implant-Related Osteomyelitis of the Jaws: An Insidious and Calamitous Entity. J Oral Maxillofac Surg 2018;76: 1187-93.

12. Rokadiya S, Malden NJ. An implant periapical lesion leading to acute osteomyelitis with isolation of Staphylococcus aureus. Br Dent J 2008;205:489-91.

13. Waasdorp JA, Evian $\mathrm{Cl}$, Mandracchia M. Immediate Placement of Implants Into Infected Sites: A Systematic Review of the Literature. J Periodontol 2010;81:801-8.

14. Balanger M, Hinet M, Vacher C, Bellaiche N, Charrier J-L, Millot S. Osteomyelitis of the Mandible after Dental Implants in an Immunocompetent Patient. Case Rep Dent 2017;2017: 9525893-5.
15. Kim YK, Choe GY, Lee DH, Yun PY. Chronic Refractory Osteomyelitis of the Mandible and Maxilla Related to Dental Implant Placement. Asian J Oral Maxillofac Surg 2008;20: 189-92.

16. Baur DA, Altay MA, Flores-Hidalgo A, Ort Y, Quereshy FA. Chronic Osteomyelitis of the Mandible: Diagnosis and Management-An Institution's Experience Over 7 Years. J Oral Maxillofac Surg 2015;73:655-65.

17. Kim S-G, Jang H-S. Treatment of chronic osteomyelitis in Korea. Oral Surg Oral Med Oral Pathol Oral Radiol Endod 2001;92: 394-8.

18. Martos-Fernández M, Saez-Barba M, López-López J, EstrugoDevesa A, Balibrea-del-Castillo JM, Bescós-Atín C. Pentoxifylline, tocopherol, and clodronate for the treatment of mandibular osteoradionecrosis: a systematic review. Oral Surg Oral Med Oral Pathol Oral Radiol 2018;125:431-9.

19. Seo MH, Eo MY, Myoung H, Kim SM, Lee JH. The effects of pentoxifylline and tocopherol in jaw osteomyelitis. J Korean Assoc Oral Maxillofac Surg 2020;46:19-27.

20. Pigrau C, Almirante B, Rodriguez D, Larrosa N, Bescos S, Raspall G, et al. Osteomyelitis of the jaw: resistance to clindamycin in patients with prior antibiotics exposure. Eur J Clin Microbiol Infect Dis 2009;28:317-23.

21. Gaetti-Jardim Júnior E, Fardin AC, Gaetti-Jardim EC, de Castro AL, Schweitzer CM, Avila-Campos MJ. Microbiota associated with chronic osteomyelitis of the jaws. Braz J Microbiol 2010;41:1056-64.

22. Kim Y, Jung H-I, Kim Y-K, Ku J-K. Histologic analysis of osteonecrosis of the jaw according to the different aspects on quantitative light-induced fluorescence images. Photodiagnosis Photodyn Ther 2021;34:102212-.

23. Kim I-h, Kim Y, Choi J, Ku J-K. The novel application of quantitative light-induced fluorescence to oral mucosal necrosis: A case report with histologic findings. Photodiagnosis Photodyn Ther 2020;31:101806-. 